\title{
BACKWARDS UNIQUENESS OF THE MEAN CURVATURE FLOW
}

\author{
HONG HUANG
}

\begin{abstract}
In this note we prove the backwards uniqueness of the mean curvature flow for (codimension one) hypersurfaces in a Euclidean space. More precisely, let $F_{t}, \widetilde{F}_{t}: M^{n} \rightarrow \mathbb{R}^{n+1}$ be two complete solutions of the mean curvature flow on $M^{n} \times[0, T]$ with bounded second fundamental forms. Suppose $F_{T}=\widetilde{F}_{T}$, then $F_{t}=\widetilde{F}_{t}$ on $M^{n} \times[0, T]$. This is an analog of a result of Kotschwar on the Ricci flow.
\end{abstract}

\section{INTRODUCTION}

In [K1] Kotschwar proved backwards uniqueness of the Ricci flow by reducing the problem to one for a suitable system of differential inequalities. Inspired by his work we prove the backwards uniqueness of the mean curvature flow for (codimension one) hypersurfaces in a Euclidean space. More precisely, we have the following

Theorem Let $F_{t}, \widetilde{F}_{t}: M^{n} \rightarrow \mathbb{R}^{n+1}$ be two complete solutions of the mean curvature flow on $M^{n} \times[0, T]$ with bounded second fundamental forms. Suppose $F_{T}=\widetilde{F}_{T}$, then $F_{t}=\widetilde{F}_{t}$ on $M^{n} \times[0, T]$.

Note that the (forward) uniqueness of the mean curvature flow in any codimension (and with more general ambient spaces) was established by Chen and Yin [CY].

As an immediate consequence of our theorem we have the following

Corollary Let $F_{t}: M^{n} \rightarrow \mathbb{R}^{n+1}$ be a complete solution of the mean curvature flow on $M^{n} \times[0, T]$ with bounded second fundamental form. Let $g_{t}$ be the induced metric on $M^{n}$ via $F_{t}$. Suppose $\sigma$ is an isometry of $\left(M^{n}, g_{T}\right)$ such that there is a Euclidean isometry $\bar{\sigma}$ of $\mathbb{R}^{n+1}$ satisfying $\bar{\sigma} \circ F_{T}=F_{T} \circ \sigma$. Then there holds $\bar{\sigma} \circ F_{t}=F_{t} \circ \sigma$ on $M^{n} \times[0, T]$.

Proof of Corollary. Note that $\bar{\sigma} \circ F_{t}$ and $F_{t} \circ \sigma$ are two solutions to the mean curvature flow on $M^{n} \times[0, T]$ with bounded second fundamental forms and with the same terminal value, so by our theorem $\bar{\sigma} \circ F_{t}=F_{t} \circ \sigma$ on $M^{n} \times[0, T]$.

In the next section we will give the proof of our theorem, which relies heavily on the methods and results in [K1] (see also [K2]). In particular, we'll use Theorem 3.1 in [K1]. We first reduce the proof of our theorem to that of the orientable case,

1991 Mathematics Subject Classification. 53C44.

Key words and phrases. mean curvature flow, backwards uniqueness, second fundamental form. 
so we can use the scalar-valued second fundamental forms instead of the vectorvalued forms. It is more convenient to use the scalar-valued second fundamental forms when we do some computations to compare two immersions of $M^{n}$ in $\mathbb{R}^{n+1}$. But towards the end of the proof we need some extra effort: We'll use the classical (Bonnet's) uniqueness theorem for hypersurfaces in a Euclidean space and ChenYin's uniqueness theorem for the mean curvature flow.

\section{Proof of Theorem}

To prove the Theorem we first note that we can assume that the manifold $M^{n}$ is connected, otherwise we can deal with each component of $M^{n}$. Furthermore we can assume that $M^{n}$ is orientable. The reason is as follows. If $M^{n}$ is not orientable, we consider the orientation double cover $p: \hat{M} \rightarrow M$. Let a family of immersions $F_{t}: M^{n} \rightarrow \mathbb{R}^{n+1}(t \in[0, T])$ be a solution to the mean curvature flow

$$
\frac{\partial}{\partial t} F(x, t)=\vec{H}(x, t)
$$

where $\vec{H}(x, t)=\vec{H}_{F}(x, t)$ is the mean curvature vector of the immersion $F_{t}=F(\cdot, t)$ at the point $x \in M$. Let $\hat{F}(\hat{x}, t)=F(p(\hat{x}), t)$ for $\hat{x} \in \hat{M}$ and $t \in[0, T]$. Then

$$
\frac{\partial}{\partial t} \hat{F}(\hat{x}, t)=\frac{\partial}{\partial t} F(p(\hat{x}), t)=\vec{H}_{F}(p(\hat{x}), t)=\vec{H}_{\hat{F}}(\hat{x}, t) .
$$

That is, $\hat{F}_{t}=\hat{F}(\cdot, t): \hat{M} \rightarrow \mathbb{R}^{n+1}$ is also a solution to the mean curvature flow, and the proof of the Theorem in the nonorientable case is reduced to that in the orientable case.

Now let $M^{n}$ be a connected, orientable, and smooth manifold, and let a family of immersions $F_{t}: M^{n} \rightarrow \mathbb{R}^{n+1}(t \in[0, T])$ be a solution to the mean curvature flow. Choose a (global) smooth, unit normal vector field $\nu$ of the immersion $F_{t}$, and write $\vec{H}=H \nu$, where $H$ is the scalar mean curvature. Let $A=\left(h_{i j}\right)$ be the (scalar) second fundamental form of the immersion $F_{t}$ w.r.t. $\nu, g=g_{t}$ be the induced metric on $M^{n}$ via $F_{t}, \nabla$ be the Levi-Civita connection of $\left(M^{n}, g_{t}\right)$, and $\Gamma_{j k}^{i}$ be the corresponding Christoffel symbols. Note that $H=g^{i j} h_{i j}$, where $\left(g^{i j}\right)$ is the inverse of the metric matrix $\left(g_{i j}\right)$.

We have the following lemma, most of which can be found in Huisken $[\mathrm{H}]$. 
Lemma 1 Along the mean curvature flow we have

$$
\begin{aligned}
\frac{\partial}{\partial t} g_{i j} & =-2 H h_{i j} . \\
\frac{\partial}{\partial t} \Gamma_{j k}^{i} & =-g^{i l}\left[\nabla_{j}\left(H h_{k l}\right)+\nabla_{k}\left(H h_{j l}\right)-\nabla_{l}\left(H h_{j k}\right)\right] . \\
\frac{\partial}{\partial t} h_{i j} & =\Delta h_{i j}-2 H h_{i l} g^{l m} h_{m j}+|A|^{2} h_{i j} . \\
\frac{\partial}{\partial t} \nabla_{k} h_{i j} & =\Delta \nabla_{k} h_{i j}+g^{p q} g^{r l}\left[2\left(h_{k i} h_{q l}-h_{k l} h_{q i}\right) \nabla_{p} h_{r j}\right. \\
& +2\left(h_{k j} h_{q l}-h_{k l} h_{q j}\right) \nabla_{p} h_{i r}+\left(h_{k q} h_{p l}-h_{k l} h_{p q}\right) \nabla_{r} h_{i j} \\
& \left.+h_{i r} \nabla_{p}\left(h_{k j} h_{q l}-h_{k l} h_{q j}\right)+h_{r j} \nabla_{p}\left(h_{k i} h_{q l}-h_{k l} h_{q i}\right)\right] \\
& +g^{l m}\left[h_{i l}\left(\nabla_{j}\left(H h_{k m}\right)-\nabla_{m}\left(H h_{k j}\right)\right)+h_{l j}\left(\nabla_{i}\left(H h_{k m}\right)\right.\right. \\
& \left.\left.-\nabla_{m}\left(H h_{k i}\right)\right)-H\left(h_{i l} \nabla_{k} h_{m j}+h_{j l} \nabla_{k} h_{m i}\right)\right] \\
& +\nabla_{k}\left(|A|^{2} h_{i j}\right) .
\end{aligned}
$$

Proof. For (2.1)-(2.3) see [H]. (2.4) follows ( by a tedious computation) from (2.2), (2.3), commutation formulas for derivatives and the Gauss equation.

Actually in this note we only need a rough form of the formula (2.4).

Now let $f=g-\widetilde{g}, P=\nabla-\widetilde{\nabla}, Q=\nabla P, S=A-\widetilde{A}$, and $U=\nabla A-\widetilde{\nabla} \widetilde{A}$, where $\widetilde{g}, \widetilde{\nabla}$, etc are the corresponding quantities w.r.t. another family of immersions $\widetilde{F}_{t}: M^{n} \rightarrow \mathbb{R}^{n+1}(t \in[0, T])$ which is also a solution to the mean curvature flow. Then we have the following

Lemma 2 Let $F_{t}$ and $\widetilde{F}_{t}$ be as above. We have

$$
\begin{aligned}
& \frac{\partial f}{\partial t}=\widetilde{g}^{-1} * f * \widetilde{A} * \widetilde{A}+S * \widetilde{A}+A * S, \\
& \frac{\partial P}{\partial t}=\widetilde{g}^{-1} * f * \widetilde{g}^{-1} * \widetilde{A} * \widetilde{\nabla} \widetilde{A}+\widetilde{g}^{-1} * f * \widetilde{A} * \widetilde{\nabla} \widetilde{A}+S * \widetilde{\nabla} \widetilde{A}+A * U, \\
& \frac{\partial Q}{\partial t}=\widetilde{g}^{-1} * P * f * \widetilde{g}^{-1} * \widetilde{A} * \widetilde{\nabla} \widetilde{A}+\widetilde{g}^{-1} * \widetilde{g} * P * \widetilde{g}^{-1} * \widetilde{A} * \widetilde{\nabla} \widetilde{A} \\
& +\widetilde{g}^{-1} * f * \widetilde{g}^{-1} * \widetilde{\nabla} \widetilde{A} * \widetilde{\nabla} \widetilde{A}+\widetilde{g}^{-1} * f * \widetilde{g}^{-1} * \widetilde{A} * \widetilde{\nabla}^{2} \widetilde{A} \\
& +\quad P * \widetilde{g}^{-1} * f * \widetilde{A} * \widetilde{\nabla} \widetilde{A}+\widetilde{g}^{-1} * \widetilde{g} * P * \widetilde{A} * \widetilde{\nabla} \widetilde{A}+\widetilde{g}^{-1} * f * \widetilde{\nabla} \widetilde{A} * \widetilde{\nabla} \widetilde{A} \\
& +\widetilde{g}^{-1} * f * \widetilde{A} * \widetilde{\nabla}^{2} \widetilde{A}+\nabla S * \widetilde{\nabla} \widetilde{A}+S * P * \widetilde{\nabla} \widetilde{A} \\
& +S * \widetilde{\nabla}^{2} \widetilde{A}+\nabla A * U+A * \nabla U+A * \nabla A * P, \\
& \left(\frac{\partial}{\partial t} \quad-\Delta\right) S=f * \widetilde{g}^{-1} * \widetilde{\nabla}^{2} \widetilde{A}+P * \widetilde{\nabla} \widetilde{A}+Q * \widetilde{A}+P * P * \widetilde{A} \\
& +\widetilde{g}^{-1} * f * \widetilde{g}^{-1} * \widetilde{A} * \widetilde{A} * \widetilde{A}+\widetilde{g}^{-1} * f * \widetilde{A} * \widetilde{A} * \widetilde{A}+S * \widetilde{A} * \widetilde{A} \\
& +A * S * \widetilde{A}+A * A * S, \\
& \left(\frac{\partial}{\partial t} \quad-\Delta\right) U=f * \widetilde{g}^{-1} * \widetilde{\nabla}^{3} \widetilde{A}+P * \widetilde{\nabla}^{2} \widetilde{A}+Q * \widetilde{\nabla} \widetilde{A}+P * P * \widetilde{\nabla} \widetilde{A} \\
& +\widetilde{g}^{-1} * \widetilde{g}^{-1} * f * \widetilde{A} * \widetilde{A} * \widetilde{\nabla} \widetilde{A}+\widetilde{g}^{-1} * f * \widetilde{A} * \widetilde{A} * \widetilde{\nabla} \widetilde{A} \\
& +\quad S * \widetilde{A} * \widetilde{\nabla} \widetilde{A}+A * S * \widetilde{\nabla} \widetilde{A}+A * A * U \text {. }
\end{aligned}
$$


(Here $V * W$ denotes a linear combination of contractions of the tensor fields $V$ and $W$ by the metric $g$.)

Proof. As in [K1], it is easy to verify that

$$
\begin{gathered}
\widetilde{g}^{-1}-g^{-1}=\widetilde{g}^{-1} * f, \\
\nabla f=\widetilde{g} * P, \\
\nabla \widetilde{g}^{-1}=(\nabla-\widetilde{\nabla}) \widetilde{g}^{-1}=\widetilde{g}^{-1} * P, \\
\widetilde{\nabla} W=\nabla W+P * W
\end{gathered}
$$

for any tensor field $W$,

$$
\widetilde{\Delta} \widetilde{A}=\Delta \widetilde{A}+f * \widetilde{g}^{-1} * \widetilde{\nabla}^{2} \widetilde{A}+P * \widetilde{\nabla} \widetilde{A}+Q * \widetilde{A}+P * P * \widetilde{A}
$$

and

$$
\widetilde{\Delta} \widetilde{\nabla} \widetilde{A}=\Delta \widetilde{\nabla} \widetilde{A}+f * \widetilde{g}^{-1} * \widetilde{\nabla}^{3} \widetilde{A}+P * \widetilde{\nabla}^{2} \widetilde{A}+Q * \widetilde{\nabla} \widetilde{A}+P * P * \widetilde{\nabla} \widetilde{A}
$$

Recall also that

$$
\frac{\partial}{\partial t} \nabla P=\nabla \frac{\partial P}{\partial t}+\frac{\partial \Gamma}{\partial t} * P
$$

Then Lemma 2 follows from Lemma 1 by direct computations.

Now as in [K1] we let

$$
\mathcal{X}=T_{2}(M) \bigoplus T_{3}(M), \quad \mathcal{Y}=T_{2}(M) \bigoplus T_{2}^{1}(M) \bigoplus T_{3}^{1}(M),
$$

and for each $t \in[0, T]$ let

$$
\mathbf{X}(t)=S(t) \bigoplus U(t) \in \mathcal{X}, \quad \mathbf{Y}(t)=f(t) \bigoplus P(t) \bigoplus Q(t) \in \mathcal{Y}
$$

where $S, U, f, P$ and $Q$ are defined as above.

Then we have the following

Lemma 3 Assume that the manifold $M^{n}$ is orientable. Let $F_{t}, \widetilde{F}_{t}: M^{n} \rightarrow \mathbb{R}^{n+1}$ be two complete solutions of the mean curvature flow on $M^{n} \times[0, T]$ with $|A|_{g_{t}} \leq K$ and $|\widetilde{A}|_{\widetilde{g}_{t}} \leq \widetilde{K}$ for some constants $K$ and $\widetilde{K}$. Suppose $F_{T}=\widetilde{F}_{T}$. Then for any $0<\delta<T$, there exists a positive constant $C=C(\delta, K, \widetilde{K}, T)$ such that

$$
\begin{aligned}
\left|\left(\frac{\partial}{\partial t}-\Delta_{g_{t}}\right) \mathbf{X}\right|_{g_{t}}^{2} & \leq C\left(|\mathbf{X}|_{g_{t}}^{2}+|\mathbf{Y}|_{g_{t}}^{2}\right) \\
\left|\frac{\partial}{\partial t} \mathbf{Y}\right|_{g_{t}}^{2} & \leq C\left(|\mathbf{X}|_{g_{t}}^{2}+|\nabla \mathbf{X}|_{g_{t}}^{2}+|\mathbf{Y}|_{g_{t}}^{2}\right)
\end{aligned}
$$

Proof. By Ecker-Huisken [EH] there exist constants $C_{m}=C_{m}(\delta, K, T)$ and $\widetilde{C}_{m}=\widetilde{C}_{m}(\delta, \widetilde{K}, T)$ such that $\left|\nabla^{m} A\right|_{g_{t}} \leq C_{m}$ and $\left|\widetilde{\nabla}^{m} \widetilde{A}\right|_{\tilde{g}_{t}} \leq \widetilde{C}_{m}$ on $M^{n} \times[\delta, T]$.

Since $|A|_{g_{t}} \leq K$, it follows from Lemma 1 (2.1) that the metrics $\left\{g_{t}\right\}_{t \in[0, T]}$ are uniformly equivalent. Similarly, the metrics $\left\{\widetilde{g}_{t}\right\}_{t \in[0, T]}$ are uniformly equivalent too. But by our assumption $F_{T}=\widetilde{F}_{T}$, and $g_{T}=\widetilde{g}_{T}$, so $\left\{g_{t}\right\}_{t \in[0, T]}$ and $\left\{\widetilde{g}_{t}\right\}_{t \in[0, T]}$ 
are equivalent to each other. It follows that $\left|\widetilde{g}^{-1}\right|_{g_{t}},\left|\widetilde{\nabla}^{m} \widetilde{A}\right|_{g_{t}},|f|_{g_{t}},|S|_{g_{t}}$, and $|U|_{g_{t}}$ are bounded.

Now we see that $|P|_{g_{t}}$ is bounded by using the second formula in Lemma 2 and the assumption $P(T)=0$. In fact, for any $x \in M^{n}$,

$$
|P(x, t)|_{g_{t}}=|P(x, T)-P(x, t)|_{g_{t}} \leq \int_{t}^{T}\left|\frac{\partial P}{\partial t}(x, s)\right|_{g_{t}} d s \leq C^{\prime} .
$$

(One can also prove this using Lemma 1 (2.2). Compare with [K1].)

Similarly $Q$ and $\nabla^{m} P$ are bounded. Then Lemma 3 follows from Lemma 2.

Now as above, let $F_{t}, \widetilde{F}_{t}: M^{n} \rightarrow \mathbb{R}^{n+1}$ be two complete solutions of the mean curvature flow on $M^{n} \times[0, T]$ with bounded second fundamental forms, where $M^{n}$ is connected and orientable. Suppose $F_{T}=\widetilde{F}_{T}$.

Using the identity

$$
\nabla^{m} \widetilde{\nabla}^{l} \widetilde{A}=\nabla^{m-1} \widetilde{\nabla}^{l+1} \widetilde{A}+\sum_{i=0}^{m-1} \nabla^{i} P * \nabla^{m-1-i} \widetilde{\nabla}^{l} \widetilde{A}
$$

one sees that $\nabla S=\nabla A-\nabla \widetilde{A}$ and $\nabla U=\nabla^{2} A-\nabla \widetilde{\nabla} \widetilde{A}$ are bounded on $M^{n} \times[\delta, T]$ for any $0<\delta<T$. So the required growth condition of [K1, Theorem 3.1] is verified.

With the help of Lemma 3, we can apply [K1,Theorem 3.1] to conclude that $\mathbf{X}=0, \mathbf{Y}=0$ on $M^{n} \times[\delta, T]$ for any $0<\delta<T$. Then by the uniqueness theorem for hypersurfaces in a Euclidean space (see for example Theorem 6.4 in Chapter VII of $[\mathrm{KN}]$ ), for each $t \in[\delta, T], F_{t}$ and $\widetilde{F}_{t}$ coincide up to an ambient Euclidean isometry. In particular, there exists a Euclidean isometry $\bar{\sigma}: \mathbb{R}^{n+1} \rightarrow \mathbb{R}^{n+1}$ such that $\bar{\sigma} \circ F_{\delta}=\widetilde{F}_{\delta}$.

Now $\bar{\sigma} \circ F_{t}$ and $\widetilde{F}_{t}$ are two complete solutions of the mean curvature flow on $M^{n} \times[\delta, T]$ with bounded second fundamental forms and with the same initial value. By Chen-Yin's uniqueness theorem for the mean curvature flow $[\mathrm{CY}], \bar{\sigma} \circ F_{t}=\widetilde{F}_{t}$ for any $t \in[\delta, T]$. In particular, $\bar{\sigma} \circ F_{T}=\widetilde{F}_{T}$. Combining with our assumption we get $\bar{\sigma} \circ F_{T}=F_{T}$. It follows that either $\bar{\sigma}=I d$ or the image of $F_{T}$ is a hyperplane in $\mathbb{R}^{n+1}$ and $\bar{\sigma}$ is a reflection w.r.t. it. In the latter case, by using what we have proved in the previous paragraph with $\widetilde{F}_{t}$ there replaced by the trivial hyperplane solution to the mean curvature flow, we see that the image of $F_{t}$ is also a hyperplane for any $t \in[\delta, T]$. So in both cases $F_{t}=\widetilde{F}_{t}$ for any $t \in[\delta, T]$. Since $\delta \in(0, T)$ can be arbitrarily small, by continuity the Theorem is proved.

Acknowledgements I was partially supported by NSFC no.10671018 and by Laboratory of Mathematics and Complex Systems, Ministry of Education, at BNU. The first version of this note was posted on the arXiv in 2009. Recently there appeared two papers extending our result above to the higher codimension case, cf. [LM] (where the ambient spaces may be certain general Riemannian manifolds) and [Z] (where the ambient spaces are Euclidean). I would like to thank the authors of these two papers for their comments on the first version of my note, in particular, thank Man-Chun Lee for pointing out a gap in the argument in it. I would also like to thank the referee for the comments.

\section{References}


[CY] B.-L. Chen, L. Yin, Uniqueness and pseudolocality theorems of the mean curvature flow, Comm. Anal. Geom. 15 (2007), no. 3, 435-490.

[EH] K. Ecker, G. Huisken, Interior estimates for hypersurfaces moving by mean curvature, Invent. Math. 105 (1991), 547-569.

[H] G. Huisken, Flow by mean curvature of convex surfaces into spheres, J. Diff. Geom. 20 (1984), no. 1, 237-266.

[KN] S. Kobayashi, K. Nomizu, Foundations of differential geometry. Vol. II. John Wiley \& Sons, Inc., 1996.

[K1] B. Kotschwar, Backwards uniqueness of the Ricci flow, Inter. Math. Res. Not. 2010, no. 21, 4064-4097.

[K2] B. Kotschwar, A short proof of backward uniqueness for some geometric evolution equations, Internat. J. Math. 27 (2016), no. 12, 1650102, 17 pp.

[LM] M.-C. Lee, M. S. John Ma, Uniqueness Theorem for non-compact mean curvature flow with possibly unbounded curvatures, arXiv:1709.00253.

[Z] Z.-H. Zhang, A note on the backwards uniqueness of mean curvature flow, Sci. China Math. (2018). https://doi.org/10.1007/s11425-017-9231-4. arXiv:1709.00798.

School of Mathematical Sciences, Beijing Normal University, Laboratory of Mathematics and Complex Systems, Ministry of Education, Beijing 100875, P.R. China

E-mail address: hhuang@bnu.edu.cn 